\title{
A Sparse Reconstruction Direct Position Determination Method based on Temporal Information Fusion
}

\author{
Yanqing Ren ${ }^{1+}$, Bin Ba ${ }^{1}$, Zhiyu Lu ${ }^{1}$ and Daming Wang ${ }^{1}$ \\ ${ }^{1}$ National Digital Switching System Engineering and Technology Research Center, Zhengzhou, China
}

\begin{abstract}
The subspace data fusion direct position determination method is unable to locate coherent signals, and suffers location accuracy loss and source resolution degradation under scenarios of low SNR, snapshot deficiency. A sparse reconstruction direct position determination method based on temporal information fusion is proposed to overcome the above-mentioned shortcomings. Firstly, the sparse representation direct position determination model is established based on the sparsity of signal distribution. Then the multi-snapshot reconstruction method is developed based on the idea of temporal information fusion. Finally, the signal source positions are estimated according to one-to-one correspondence between the sparse vector and signal geographic-grid locations. The simulations indicate that the proposed method is able to locate coherent signals and outperforms subspace data fusion method in terms of location accuracy and source resolution.
\end{abstract}

Keywords: Direct Position Determination, Sparse Reconstruction, Temporal Information Fusion, Array Signal Processing

\section{Introduction}

The most common methods for position determination of radio signal emitters in passive location system are based on two-step processing. Firstly, specified parameters such as time of arrival (TOA) or direction of arrival (DOA) are estimated from received source signals. Next, equations of LOP including former estimated parameters are established to determine the locations of signal transmitters $[1,2]$. The two-step methods separate parameter estimation and position calculation, leading to position information loss and parameter-location mismatch. Therefore, these methods are sub-optimal. Direct position determination (DPD) method estimates the source positions directly from raw outputs without computing intermediate parameters. They avoids location information loss and can gain higher location accuracy [3-6].

There are three kinds of methods to solve the direct position determination: Maximum Likelihood methods (ML) [7-10], intelligent optimization methods [11] and Subspace Data Fusion (SDF) methods [12, 13]. Weiss A. J. firstly proposed the single-target direct position determination method based on the ML criterion [7]. However, ML-DPD methods require a multidimensional search in the presence of multiple transmitters with huge computation. Oispuu $\mathrm{M}$ and Nickel $\mathrm{U}$ transformed the multidimensional search into several low dimensional search by alternating projection to reduce computation [9]. While location accuracy is susceptible to initial value selection. Quantum behaved Particle Swarm Optimization DPD (QPSO-DPD) was proposed in [11] to reduce computation significantly. However, selecting the control parameters difficultly increases the possibility of trapping in local optimum. SDF-DPD was proposed to solve multitarget location problem in single moving station case [12], based on the ideas of multiple signals classification (MUSIC). Then [13] proposed a stationary multi-station DPD method with the theory of MUSIC. SDF-DPD method reduces complexity of multi-target DPD and have a good resolution.

\footnotetext{
+ Corresponding author. Tel.: + 8618939252755.

E-mail address: yq_renice@163.com.
} 
Unfortunately, coherent signals often exist due to the complexity of signal propagation environment and SDF-DPD methods can not locate coherent signals efficiently. Moreover, statistical characteristic of sampling covariance matrix is insufficient under the snapshot deficiency condition, which causes location accuracy of the SDF-DPD methods loss and source resolution degradation. Sparse Reconstruction (RS) idea arised recently maybe provide a new way to overcome the above-mentioned shortcomings. It need not use the statistical characteristics of the covariance matrix and can locate coherent signal sources efficiently. It has been widely used in parameter estimation such as TOAs and DOAs [14, [15].

The objective of this study is to propose a new DPD method (denoted by TSR-DPD) that can locate coherent signal sources and improve high location accuracy and source resolution under low signal-to-noise ratio (SNR) and snapshot deficiency conditions. The proposed method solves the location problem with the theory of sparse reconstruction. We establish the sparse representation DPD model at first. Then the sparse vector is reconstructed using multi-snapshot matching pursuit method based on the idea of temporal information fusion. Finally, source positions are estimated from the sparse vector. Simulations indicate that the proposed method can locate coherent signal sources efficiently. It also has higher location accuracy and source resolution than SDF-DPD method under low SNR and snapshot deficiency conditions.

The rest of this paper is organized as follows. The following section gives the DPD model. Section 3 establishes sparse representation model of DPD and develops a sparse reconstruction method based on temporal information fusion. In section 4, we derive the model CRLB. Simulation results are provided in section 5. Section 6 concludes the paper.

The following notations are used. $\mathrm{E}\{*\}$ denotes the statistical expectation operation, $(*)^{T}$ denotes transpose, $(*)^{H}$ denotes conjugate transpose, $\phi$ denotes empty set of real numbers, $\boldsymbol{\Phi}$ denotes empty set of vectors, $\operatorname{Re}\{*\}$ denotes the real part of $*$ and $\operatorname{Re}\{*\}$ represents the imaginary part of $*$.

\section{Problem Formulation}

Consider $Q$ transmitters located at positions $\boldsymbol{p}_{q}=\left(x_{q}, y_{q}\right)^{T}$ for $q=1, \cdots, Q$, and $L$ base stations intercepting the transmitted signals. The bandwidth of the signals is small compared to the inverse of the propagation time over each array aperture. Each base station is equipped with a uniform linear array (ULA) consisting of $M$ elements. The complex envelopes of signals observed by the $l$ th base station array, located at positions $\left(x_{l}, y_{l}\right)$ for $l=1, \cdots, L$, are given by the time-dependent $M \times 1$ vector

$$
\begin{aligned}
\boldsymbol{r}_{l}(t)= & \sum_{q=1}^{Q} \beta_{l, q} \boldsymbol{a}_{l}\left(\boldsymbol{p}_{q}\right) s_{q}(t)+\boldsymbol{n}_{l}(t), \quad 0 \leq t \leq T, \\
& \left\{\begin{array}{l}
\boldsymbol{a}_{l}\left(\boldsymbol{p}_{q}\right)=\left[1, \mathrm{e}^{-j \frac{2 \pi}{\lambda} d \sin \theta_{q}}, \cdots, \mathrm{e}^{-j \frac{2 \pi}{\lambda}(M-1) d \sin \theta_{q}}\right]^{T}, \\
\theta_{q}=\arctan \left(\frac{y_{q}-y_{l}}{x_{q}-x_{l}}\right), q=1, \cdots, Q ; l=1, \cdots, L,
\end{array}\right.
\end{aligned}
$$

where $\beta_{l, q}$ is complex amplitude between the $q$ th transmitter and the $l$ th base station and obeys Hata propagation attenuation model. $\boldsymbol{a}_{l}\left(\boldsymbol{p}_{q}\right)$ is the $l$ th array response to a transmitter from $\boldsymbol{p}_{q}$, and $s_{q}(t)$ is the $q$ th unknown signal waveform. The vector $\boldsymbol{n}_{l}(t)$ represents white, zero-mean, complex Gaussian noise. $T$ represents observation interval. In matrix notation, (1) becomes

$$
\boldsymbol{r}_{l}(t)=\boldsymbol{A}_{l}(\boldsymbol{p}) \boldsymbol{s}(t)+\boldsymbol{n}_{l}(t)
$$

where

$$
\left\{\begin{array}{l}
\boldsymbol{A}_{l}(\boldsymbol{p})=\left[\boldsymbol{\beta}_{l, 1} \boldsymbol{a}_{l}\left(\boldsymbol{p}_{1}\right), \ldots, \boldsymbol{\beta}_{l, Q} \boldsymbol{a}_{l}\left(\boldsymbol{p}_{Q}\right)\right], \\
\boldsymbol{s}(t)=\left[s_{1}(t), \ldots, s_{Q}(t)\right]^{T},
\end{array}\right.
$$

Concatenate the observed vectors at all base stations and form the following equation that encompasses all the raw data of the location system

where

$$
\boldsymbol{r}(t)=\boldsymbol{A s}(t)+\boldsymbol{n}(t)
$$




$$
\left\{\begin{array}{c}
\boldsymbol{r}(t)=\left[\boldsymbol{r}_{1}(t)^{T}, \cdots, \boldsymbol{r}_{L}(t)^{T}\right]^{T}, \\
\boldsymbol{A}=\left[\boldsymbol{A}_{1}^{T}(\boldsymbol{p}), \cdots \boldsymbol{A}_{L}^{T}(\boldsymbol{p})\right]^{T}=\left[\hat{\boldsymbol{a}}\left(\boldsymbol{p}_{1}\right), \cdots, \hat{\boldsymbol{a}}\left(\boldsymbol{p}_{Q}\right)\right], \\
\boldsymbol{n}(t)=\left[\boldsymbol{n}_{1}(t)^{T}, \cdots, \boldsymbol{n}_{L}(t)^{T}\right]^{T},
\end{array}\right.
$$

Here, $\boldsymbol{A}$ is the array response matrix.

\section{A Sparse Reconstruction DPD Method Based on Temporal Information Fusion}

The sparse representation of the DPD model is the precondition and reconstruction method design is a key step, when we locate signal sources directly using sparse reconstruction theory. In the following section, we will develop a sparse reconstruction DPD method based on temporal information fusion (TSR-DPD) from aspects of sparse DPD model building and reconstruction method design.

\subsection{Sparse DPD Model construction}

Figure 1 shows the signal source distribution in the interest area and we can divide the interest area into $K$ grids. Let $\boldsymbol{\varphi}_{k}$ denote the coordinates of the $k$ th grid point. For better insight and simpler notation, we make assumption that the different signal locations are on the grid. The shaded areas represent true locations of signal sources and the white areas represent virtual locations of signal sources. The source number $Q$ is far less than grid number $K$ in practice and the source distribution has the property of spatial sparsity.

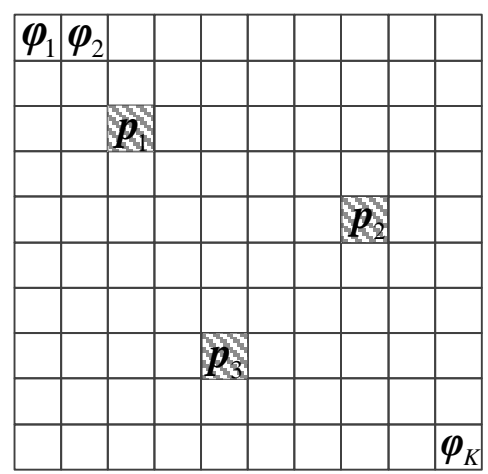

Fig. 1: Sparse distribution model of signal sources.
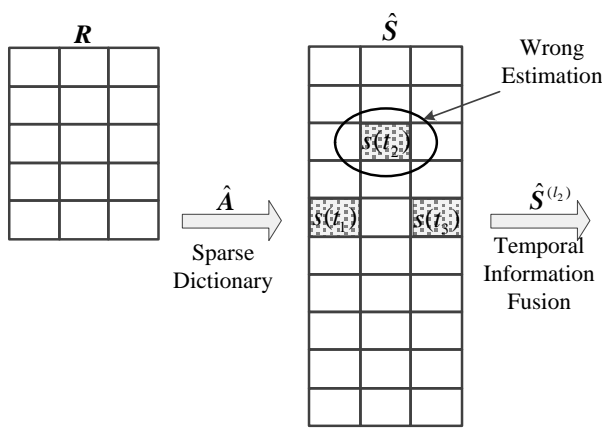

Fig. 2: The main idea of the proposed method under multi-snapshot and single target conditions.

Assume that each grid is a potential location for a true signal source, let

$$
\begin{aligned}
& \hat{\boldsymbol{A}} \triangleq\left[\hat{\boldsymbol{a}}\left(\boldsymbol{p}_{1}\right), \ldots, \hat{\boldsymbol{a}}\left(\boldsymbol{p}_{k}\right), \ldots, \hat{\boldsymbol{a}}\left(\boldsymbol{p}_{K}\right)\right] \\
& \hat{\boldsymbol{s}}(t) \triangleq\left[s_{1}(t), \ldots, s_{k}(t), \ldots, s_{K}(t)\right]^{\mathrm{T}}
\end{aligned}
$$

where

$$
s_{k}(t)=\left\{\begin{array}{c}
s_{q}(t), \boldsymbol{\varphi}_{k}=\boldsymbol{p}_{q} \\
0, \boldsymbol{\varphi}_{k} \neq \boldsymbol{p}_{q}
\end{array}\right.
$$

Therefore, equation (5) yields immediately

$$
\boldsymbol{r}(t)=\hat{\boldsymbol{A}} \hat{\boldsymbol{s}}(t)+\boldsymbol{n}(t)
$$

where $\hat{\boldsymbol{A}}$ is the sparse dictionary, $\hat{\boldsymbol{s}}$ is the sparse vector and $s_{k}(t)$ is the signal source on $k$ th grid point.

Equation (9) means that the sparse vector $\hat{\boldsymbol{s}}(t)$ has exactly $Q$ non-zero entries, and the indices (collection of all indices can be called index set) of non-zero entries correspond to the actual locations of the signals. According to the theory of sparse reconstruction, the position estimation can be transformed into $l_{0}$ norm minimization problem of equation (11):

$$
\begin{gathered}
\min \|\hat{\boldsymbol{s}}\|_{0} \\
\|\boldsymbol{r}-\hat{\boldsymbol{A}} \hat{\boldsymbol{s}}\|_{2}<\varepsilon .
\end{gathered}
$$

\subsection{Multi-snapshot Matching Pursuit Reconstruction Method Design}


The problem formulated by equation (11) is a NP-hard problem [16]. To solve the problem efficiently, it can be modeled as a linear program using matching pursuit method (MP). The main idea of the MP is to select the column vectors (atoms) from the sparse dictionary $\hat{\boldsymbol{A}}$ and realize the optimal linear representation of the observation vector $\boldsymbol{r}$. The linear representation coefficients form the sparse vector $\hat{\boldsymbol{s}}$ which corresponds to actual signal locations. We can project the residual vector onto the sparse dictionary $\hat{\boldsymbol{A}}$ and pick the column, which has the highest correlation with the residual vector, of $\hat{A}$ as the atom.

The original MP method mentioned above can only realize single-snapshot problem like equation (11), which might cause unreliable estimates. Under the large snapshot number condition, equation (5) yields

$$
\boldsymbol{R}_{M L \times J}=\boldsymbol{A}_{M L \times Q} \boldsymbol{S}_{Q \times J}+\boldsymbol{N}_{M L \times J},
$$

where

$$
\left\{\begin{array}{l}
R \triangleq[\boldsymbol{r}(1), \cdots, \boldsymbol{r}(J)], \\
S \triangleq[s(1), \cdots, s(J)], \\
N \triangleq[\boldsymbol{n}(1), \cdots, n(J)] .
\end{array}\right.
$$

The sparse DPD model the under multi-snapshot condition correspondly is

$$
\begin{aligned}
\boldsymbol{R}_{M L \times J} & =\hat{\boldsymbol{A}}_{M L \times K} \hat{\boldsymbol{S}}_{K \times J}+\boldsymbol{N}_{M L \times J}, \\
\hat{\boldsymbol{S}} & \triangleq[\hat{\boldsymbol{s}}(1), \cdots, \hat{\boldsymbol{s}}(J)],
\end{aligned}
$$

where $J$ denotes snapshot and $\hat{\boldsymbol{S}}$ represents the row-sparsity matrix. If and only if $\boldsymbol{\varphi}_{k}=\boldsymbol{p}_{q}$, entries of the $k$ th row in the row-sparsity matrix $\hat{\boldsymbol{S}}$ are not all zero. Therefore, equation (14) is modeled as a multisnapshot sparse reconstruction problem.

The sparse reconstruction problem under the multi-snapshot condition is different from it under single snapshot condition. The observation matrix $\boldsymbol{R}$ contains not only spatial information but also temporal information. While signal distribution only has the property of spatial sparsity and does not have the property of temporal sparsity. We could take temporal information fusion measure to utilize above property. Firstly, we can operate $l_{2}$ norm on temporal samples of each spatial dimension and get a spatial sparsity vector. Then we encourage spatial constraints via operating $l_{0}$ norm on the spatial sparsity vector and reconstruct sparse sparsity vector using single-snapshot MP method mentioned above. So we can convert (11) into the multisnapshot sparse reconstruction problem defined by

$$
\begin{gathered}
\min \|\hat{\boldsymbol{S}}\|_{2,0} \\
\|\boldsymbol{R}-\hat{\boldsymbol{A}} \hat{\boldsymbol{S}}\|_{F}<\varepsilon,
\end{gathered}
$$

where

$$
\|\hat{\boldsymbol{S}}\|_{u, v}=\left\|\left[\|\hat{\boldsymbol{S}}[1,:]\|_{u},\|\hat{\boldsymbol{S}}[2,:]\|_{u}, \ldots,\|\hat{\boldsymbol{S}}[K,:]\|_{u}\right]^{\mathrm{T}}\right\|_{v} .
$$

To make it easier to understand, figure 2 shows the main idea of sparse reconstruction based on temporal information fusion, under multi-snapshot and single target conditions. The row-sparsity matrix $\hat{S}$ is transformed into a sparse vector $\hat{S}^{\prime}$ via $l_{2}$ norm operation. And the maximum entry of the sparse vector $\hat{S}^{\prime}$ corresponds to the true signal position.

According to the above analysis, the main steps of the multi-snapshot matching pursuit method can be summarized as follows

1) Initialize the residual matrix $\boldsymbol{E} r r_{0}=\boldsymbol{R}$, iteration times $i=1$, index set $\Lambda_{0}=\phi$, sparse degree $Q$ and atom library $\Phi_{0}=\boldsymbol{0}$. We also compute the sparse dictionary $\hat{\boldsymbol{A}}$ at the beginning of iteration;

2) Project the residual matrix $\boldsymbol{E} \boldsymbol{E} \boldsymbol{r}_{i}$ onto the sparse dictionary $\hat{\boldsymbol{A}}$, according to the equation (18), and get projection values $\mu_{k^{\prime}, j}$ of each snapshot. Then operate $l_{2}$ norm on each row of projection matrix $\boldsymbol{U}_{i}$ composed of $\mu_{k^{\prime}, j}$ and get the projection vector $\boldsymbol{u}_{i}$ of temporal information fusion. Finally, find out index $\lambda_{i}$ of maximum element from the projection vector $\boldsymbol{u}_{i}$.

$$
\mu_{k^{\prime}, j^{\prime}}=\left\langle\left\langle\boldsymbol{E r r}_{i}\left(j^{\prime}\right), \hat{\boldsymbol{a}}\left(\boldsymbol{p}_{k^{\prime}}\right)\right\rangle\right|, k^{\prime}=1, \ldots, K, j^{\prime}=1, \ldots, J,
$$

where $\boldsymbol{E r r}_{i}\left(j^{\prime}\right)$ is $j^{\prime}$ th column of the residual matrix $\boldsymbol{E r r}_{i}$. 
3) Update index set $\Lambda_{i}=\Lambda_{i-1} \cup \lambda_{i}$ and atom library $\Phi_{i}=\Phi_{i-1} \cup \hat{\boldsymbol{a}}\left(\boldsymbol{p}_{\lambda_{i}}\right)$;

4) Update the residual matrix $\boldsymbol{E} \boldsymbol{r} \boldsymbol{r}_{i}=\boldsymbol{R}-\Phi_{i}\left(\Phi_{i}{ }^{\mathrm{T}} \Phi_{i}\right)^{-1} \Phi_{i}^{\mathrm{T}} \boldsymbol{R}$ with the principle of the least square;

5) Update iteration times $i=i+1$. If $i<Q$, return to the second step, otherwise, end iteration steps and export index set $\Lambda_{i}$.

The multi-snapshot reconstruction method takes full advantage of temporal information, which avoids unreliable estimates under single-snapshot condition. In conclusion, the main steps of our proposed method can be summarized as follows:

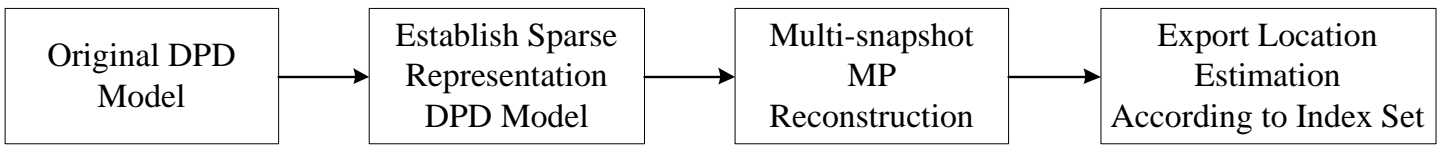

Fig. 3: Processing steps of TSR-DPD method.

\section{Numerical Results}

In the following section, we will compare TSR-DPD method with SDF-DPD method from aspects of decoherence ability, location accuracy, source resolution and computational complexity. Consider three base stations located at $(1.5,-1) \mathrm{km},(0,3) \mathrm{km}$ and $(0,0) \mathrm{km}$. Each base station is equipped with a ULA of 9 antenna elements. The spacing between elements is $\Delta=\lambda / 2$, where $\lambda$ is the wavelength of the transmitted signals. The carrier frequency of the simulated signal is assumed to be $900 \mathrm{MHz}$. Source number, source location, snapshot and SNR will be elaborated in different experiments, according to different simulation purposes.

Define SNR for the passive location system as

$$
\mathrm{SNR}=10 \lg \frac{|y(t)|^{2}}{|w(t)|^{2}},
$$

where $y(t)$ is the observed signal and $w(t)$ is additive white Gaussian noise.

Root mean square error (RMSE) is defined by

$$
\mathrm{RMSE}=\sqrt{\frac{1}{N}\left(\sum_{i=1}^{N}\left(x_{i}-\hat{x}\right)^{2}+\left(y_{i}-\hat{y}\right)^{2}\right)}
$$

where $N$ is the number of experiments, $(\hat{x}, \hat{y})$ is the emitter location, and $\left(x_{i}, y_{i}\right)$ is the $i$ th location estimate. Test Case 1: In the first experiment, we will test decoherence ability of TSR-DPD method. We used three coherent signal sources placed at $(1,3) \mathrm{km},(2,0) \mathrm{km}$ and $(4,2) \mathrm{km}$. The snapshot is 100 and SNR is $30 \mathrm{~dB}$. The results of two methods are shown in Figure 3.

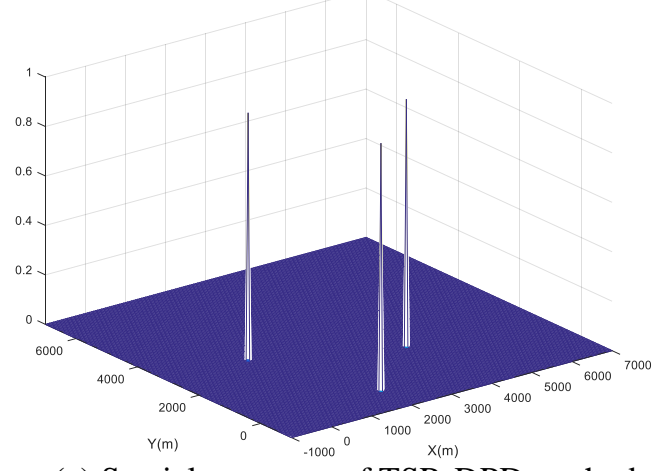

(a) Spatial spectrum of TSR-DPD method.

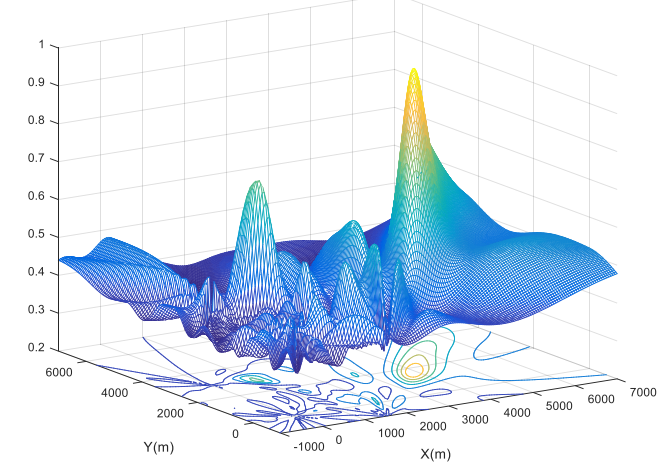

(b) Spatial spectrum of SDF-DPD method.

Fig. 3: Decoherence ability comparison between TSR-DPD method and SDF-DPD method.

As Fig 3 depicted, lots of false peaks arise in the spatial spectrum of SDF-DPD method and we can hardly distinguish true peaks around signal source positions. In contrast, three peaks around signal source positions arise clearly in the spatial spectrum of TSR-DPD method. That might be due to the fact that the covariance matrix is rank-deficient because of coherent signals and SDF-DPD method essentially belongs to subspace methods, which leads to SDF-DPD method being unable to locate coherent signal sources. While TSR-DPD method does not need the covariance matrix to locate coherent signal sources and will not face the problem of matrix rank deficiency. Therefore, TSR-DPD method can locate coherent signal sources efficiently. 
Test Case 2: In the second experiment, we shall contrast the localization performance of TSR-DPD method with SDF-DPD method under the snapshot deficiency condition. The transmitter position is $(4,2) \mathrm{km}$ and snapshot is 10 . The SNR is varied between $-12 \mathrm{~dB}$ and $8 \mathrm{~dB}$ using $2 \mathrm{~dB}$ steps. The results are obtained from 100 Monte Carlo ensemble runs and they are summarized in figure 4.

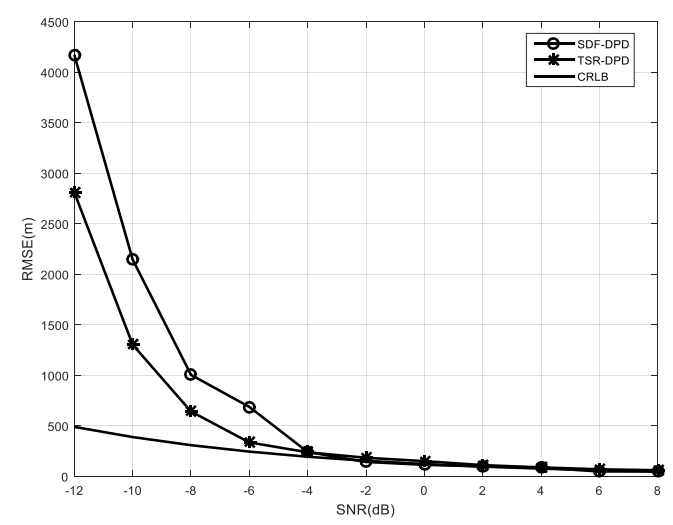

Fig. 4: RMSE of two methods under the snapshot deficiency condition.

Figure 4 shows that, compared with SDF-DPD method, location accuracy of TSR-DPD method increases by about $30 \%$, when SNR is below $-5 \mathrm{~dB}$. Both of two methods are close to the CRLB along with SNR increasing. Therefore, the proposed method has higher location accuracy than SDF-DPD method under scenarios of low SNR and snapshot deficiency.

Test Case 3: In order to compare TSR-DPD method with SDF-DPD method in terms of source resolution, under scenarios of low SNR and snapshot deficiency, two signal sources are located at $(2,2) \mathrm{km}$ and $(3,2) \mathrm{km}$. The number of snapshots is 10 and the SNR is $-5 \mathrm{~dB}$. The spatial spectrums are plotted in figure 5 .

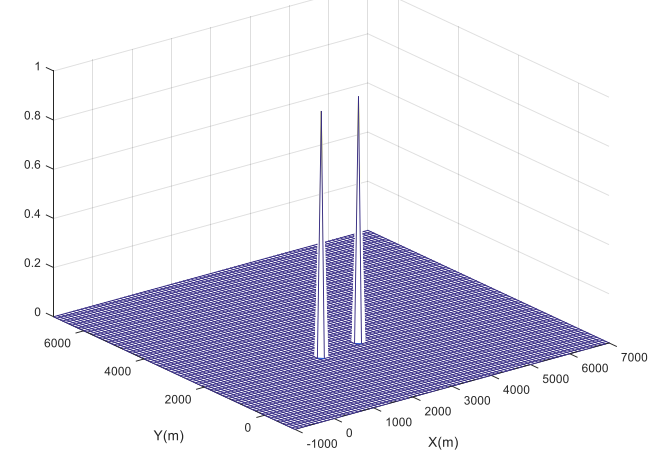

(a)TSR-DPD method spatial spectrum.

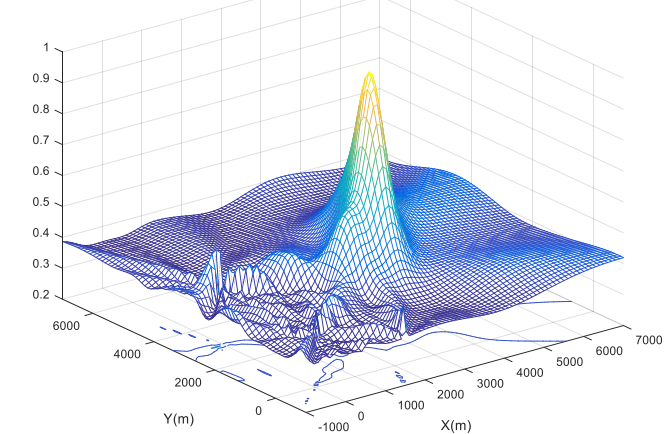

(b) SDF-DPD method spatial spectrum.

Fig. 5: Source resolution contrast between TSR-DPD method and SDF-DPD method.

As Fig. 5(a) depicted, two sharp peaks of the TSR-DPD spectrum are visible and the proposed method can distinguish two near sources clearly and correctly. In contrast, SDF-DPD method can hardly distinguish two peaks. The result indicates that TSR-DPD method has higher source resolution than SDF-DPD method under scenarios of low SNR and a few snapshots.

\section{Complexity Analysis}

The computational complexity of SDF-DPD method mainly composes three parts: covariance matrix calculation, eigen-decomposition and cost function searching. The computational complexity of TSR-DPD method also composes three parts: computing sparse dictionary, projecting the residual matrix onto the sparse dictionary and updating the residual matrix. The computational complexity of each method is shown in Table 1.

Table 1. Computational complexity comparison of two methods

\begin{tabular}{cc}
\hline Method Name & Computational Complexity \\
\hline SDF-DPD & $o\left((M L)^{2} J+(M L)^{3}+(M L)^{2}+K M L(M L-Q)\right)$
\end{tabular}


TSR-DPD

$$
o\left(\begin{array}{c}
M L K+Q K M L J+Q(Q+1) M L J \\
+\frac{M L\left(2 Q^{3}+3 Q^{2}+Q\right)}{3}+\frac{Q^{4}+2 Q^{3}+Q}{4}
\end{array}\right)
$$

Considering that station number $L$, source number $Q$ and array elements $M$ are often small, the complexity of SDF-DPD can be approximated as $o\left((M L)^{2} J+K M L(M L-Q)\right)$ and the complexity of TSRDPD can be approximated as $o(M L K+Q K M L J+Q(Q+1) M L J)$. If snapshot number is very large, TSRDPD will have heavier computational complexity than SDF-DPD. However, the complexity of TSR-DPD will be equivalent to or even lower than the complexity of SDF-DPD, if snapshot number is small. Therefore, the proposed method is an excellent method under the snapshot deficiency condition.

\section{Conclusions}

The existing SDF-DPD method, in the multi-target location system, has low complexity but cannot locate coherent signal sources efficiently. And the SDF-DPD method also has location accuracy loss and source resolution degradation under scenarios of low SNR and snapshot deficiency. The proposed method establishes a sparse representation DPD model and develops multi-snapshot matching pursuit method based on the temporal information fusion to overcome the above-mentioned shortcomings. The simulations show that the proposed DPD is able to locate coherent signal sources and also has higher location accuracy and source resolution than the SDF-DPD under scenarios of low SNR and snapshot deficiency.

\section{Acknowledgements}

This work was supported by the National Natural Science Foundation of China (No. 2012AA01A502, 2012AA01A505) and the National High Technology Research and Development Program of China (No. 61401513).

\section{References}

[1] Liu, F. Passive Location and Tracking, Xi-Dian Pub. China, 2011.

[2] Tian, X. Theory and Technology of Wireless Location, National Defense Industry Pub. China, 2011.

[3] Tzafri, L. and A. J. Weiss, "High Resolution Direct Position Determination Using MVDR" IEEE Transactions on Wireless Communications, Vol. 15, No.9, 6449-6461, 2016.

[4] Bar-Shalom, O. and A. J. Weiss, "Direct emitter geolocation under local scattering” IEEE Signal Processing, Vol. 117, 102-114, 2015.

[5] Li, J., L. Yang. and F. Guo, "Coherent summation of multiple short-time signals for direct positioning of a wideband source based on delay and Doppler,” IEEE Digital Signal Processing, Vol. 48, 58-70, 2016.

[6] Tirer, T. and A. J. Weiss, "High Resolution Direct Position Determination of Radio Frequency Sources" IEEE Signal Processing Letters, Vol. 23, No.2, 192-196, 2015.

[7] Weiss, A.J. "Direct position determination of narrowband radio frequency transmitters" IEEE Signal Processing Letters, Vol. 11, No.5, 513-516, 2004.

[8] Reuven, A.M. and A. J. Weiss, "Direct position determination of cyclostationary signals" IEEE Signal Processing, Vol. 89, No.12, 2448-2464, 2009.

[9] Oispuu, M. and U. Nickel, "Direct detection and position determination of multiple sources with intermittent emission” IEEE Signal Processing, Vol. 90, No.12, 3056-3064, 2010.

[10] Amar, A. and A. J. Weiss, “A decoupled method for geolocation of multiple emitters" IEEE Signal Processing, Vol. 87, No.10, 2348-2359, 2007.

[11] Zhang, M., F. Guo. and Y. Zhou, "A Single Moving Observer Direct Position Determination Method Using a Long Baseline Interferometer,” Journal of Aeronautics, Vol. 34, No.2, 378-386, 2013.

[12] Demissie, B., "Localization of multiple sources with a moving array using subspace data fusion," in International Conference on Information Fusion, Cologne, Germany, 2008, 1-7.

[13] Huang, Z. and J. Wu, "Multi-array Data Fusion Based Direct Position Determination Method" in International 
Symposium on Computational Intelligence \& Design, Guangzhou, China, 121-124, 2014.

[14] Li, Zhuo. and Jing, Yan. Compressive Sensing and Its Applications, National Defense Industry Pub. China, 2015.

[15] Yeredor, A. and E. Angel, "Joint TDOA and FDOA Estimation: A Conditional Bound and Its Use for Optimally Weighted Localization,” IEEE Transactions on Signal Processing, Vol. 59, No. 4, 1612-1623, 2011.

[16] Elad, M. Sparse and Redundant Representations: From Theory to Applications in Signal and Image Processing, Springer Publishing Company, Incorporated, 2010. 